\title{
Preparation and Magnetic Properties of Fe Doped Zno Thin Films with Dilute Magnetic Semiconductors
}

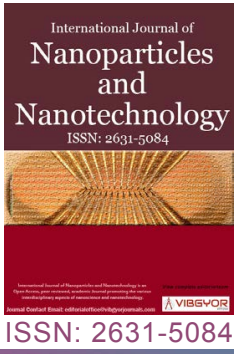

\section{Yidong Zhang*, Jing Liu and Yuanhao Gao}

Key Laboratory for Micro-Nano Energy Storage and Conversion Materials of Henan Province, Institute of Surface Micro and Nano Materials, Xuchang University, Xuchang, China

\begin{abstract}
Fe doped $\mathrm{ZnO}$ solid solution and its semiconductor thin films were prepared by sol-gel method. After aging and quenching, the films were characterized by X-ray diffraction (XRD) and atomic force microscopy (AFM). The elemental proportion was obtained by energy dispersive spectrometer (EDS) and the magnetic properties were measured by magnetic force microscope (MFM). The results show that iron doping can inhibit the growth of zinc oxide particles, and then increase the roughness of thin films. The results of MFM show that Fe doping can make non-magnetic $\mathrm{ZnO}$ semiconductors become dilute magnetic semiconductors. With the increasing amount of Fe doping, the distribution of magnetic domains and magnetic moments becomes more obvious.
\end{abstract}

\section{Keywords}

Sol-gel, Fe-doped ZnO thin films, Magnetic force microscopy (MFM), Ferromagnetism

\section{Introduction}

With the development of spintronics, diluted magnetic semiconductors (DMSs), as the main research materials, have been extensively explored. DMSs have excellent magnetic, magneto-optic and magnetoelectronic properties, and have important applications in high-density nonvolatile memory, magnetic inductors, optical isolators, semiconductor integrated circuits, semiconductor lasers and spin quantum computers [1]. Zinc oxide is a multifunctional broadband semiconductor. Its band gap at room temperature is about $3.37 \mathrm{eV}$ [2]. Because of its high melting point $\left(1975^{\circ} \mathrm{C}\right)$ and high chemical and thermal stability, zinc oxide can be doped as a matrix to obtain thin magnetic semiconductor films, while zinc oxide single crystal films can be obtained at temperatures below $500^{\circ} \mathrm{C}$ [3]. Therefore, the defects produced by high temperature preparation can be greatly reduced by high temperature quenching [4] As a wide band gap semiconductor, ZnO has shown more and more important research value and potential application value. There are many methods for preparing $\mathrm{ZnO}$, such as precipitation [5], hydrothermal [6] and electrospinning [7]. Especially, sol-gel is a very promising method with the characteristics of high purity, good uniformity, fine particle size, low synthetic temperature and simple process equipment [8].

*Corresponding author: Yidong Zhang, Key Laboratory for Micro-Nano Energy Storage and Conversion Materials of Henan Province, Institute of Surface Micro and Nano Materials, Xuchang University, Xuchang, 461000, P.R. China, Tel: +86-374-2968783

Accepted: June 06, 2019; Published: June 08, 2019

Copyright: (c) 2019 Zhang Y, et al. This is an open-access article distributed under the terms of the Creative Commons Attribution License, which permits unrestricted use, distribution, and reproduction in any medium, provided the original author and source are credited.

Zhang et al. Int J Nanoparticles Nanotech 2019, 5:028

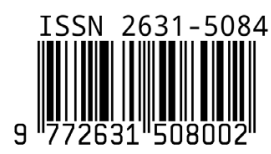


The strong exchange between carriers and local magnetic moments changes the band structure and the behavior of carriers, which makes DMSs present many new physical phenomena [9]. The theory shows that the wide bandgap semiconductor $\mathrm{ZnO}$ may have a higher Curie temperature [10], which makes the ZnO-based DMS materials become one of the research hotspots. Shinde, et al. studied the electronic structure and magnetic properties of $\mathrm{Ni}$ doped $\mathrm{ZnO}$ nanopowders. It was found that the different doping positions resulted in three different coupling states of ferromagnetism, antiferromagnetism and paramagnetism [11]. Presently, there are few theoretical studies on Fe-doped $\mathrm{ZnO}$ films [12].

In this study, EDS and XRD were used to investigate the crystal structure and the proportion of atoms. MFM was used to study the surface roughness, magnetic domains and magnetic moments of Fe-doped $\mathrm{ZnO}$ thin film.

\section{Experimental Procedure}

All the reagents used in the experiments were in analytic grade (purchased from Shanghai Sinopharm Chemical Reagent Co., Ltd) and used without further purification.

\section{Preparation of Fe-doped $\mathrm{ZnO}$ thin films}

ZnO sols were fabricated by sol-gel method using zinc acetate, anhydrous ethanol, and diethanolamine (DMA) as the solute, solvent, and sol stabilizer, respectively. In a typical procedure, zinc acetate was first dissolved in ethanol at $50{ }^{\circ} \mathrm{C}$, then DMA and a certain amount of iron acetate was slowly added into the solution under fierce stirring for an hour to form transparent Fe-doped $\mathrm{ZnO}$ mixed sols. The amount of iron acetate was controlled according to the molar ratio between $\mathrm{ZnO}$ and Fe. In the sol, the molar ratio of DMA to zinc acetate was kept at 1.0, and the $\mathrm{Zn}$ concentration was $0.5 \mathrm{M}$. Then Fe-doped $\mathrm{ZnO}$ thin films were prepared by a spin coating method on glass substrates for $30 \mathrm{sec}$ with a spinning speed of $2000 \mathrm{rpm}$. The glass substrates were ultrasonically cleaned by ethanol, acetone, and distilled water for $30 \mathrm{~min}$, respectively. After the spin coating, the resulting Fe-doped $\mathrm{ZnO}(\mathrm{x}=0$, $1,5$, and $10 \mathrm{~mol} \%)$ thin films were annealed at 500 ${ }^{\circ} \mathrm{C}$ in air for $1 \mathrm{~h}$, which are marked with $\mathrm{a}, \mathrm{b}, \mathrm{c}$ and d, respectively.

The crystal structure of Fe-doped $\mathrm{ZnO}$ thin films were determined by XRD (Bruker D8 advance; Germany) with $\mathrm{Cu}$ Ka radiation (1.54 $\AA$ ) operating at $40 \mathrm{kV}$. The surface morphology and magnetic domains of the thin films were observed by MFM, (Bruker Dimension ICON, Nanoscope V) at a scan rate of $1.0 \mathrm{~Hz}$ with commercial $\mathrm{CoCr}$ coated silicon

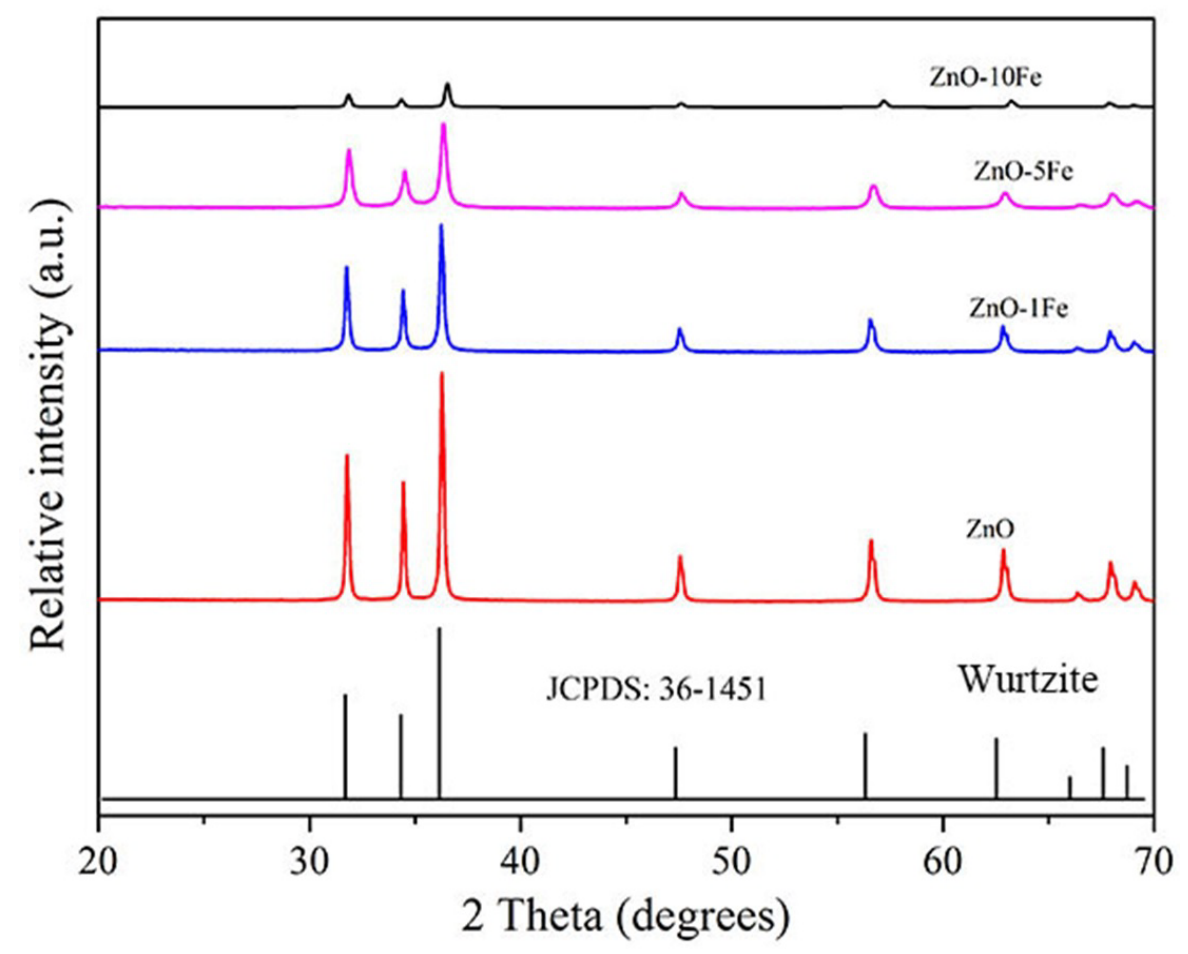

Figure 1: XRD of Fe-doped ZnO. 
probe under ambient conditions $\left(25^{\circ} \mathrm{C}\right)$. The tip radius, spring constant, and resonance frequency of the probe are less than $10 \mathrm{~nm}, 2.8 \mathrm{~N} / \mathrm{m}$, and 75 $\mathrm{kHz}$, respectively.

\section{Result and Discussion}

The XRD patterns of $\mathrm{ZnO}$ thin films with different Fe content annealed at $500{ }^{\circ} \mathrm{C}$ are shown in Figure 1 . The diffraction peaks of samples, which corresponded to (100), (002) and (101) of standard ZnO powder, indicating that the samples were polycrystalline hexagonal wurtzite structure (Zincite, JCPDS 36-1451). It can be seen that the half-peak width increases with the increase of Fe doping. According to Scheller's formula [13],

$$
D=\frac{k \lambda}{\beta \cos \theta}
$$

Where $\mathrm{k}=0.9$ is the shape factor, $\lambda$ is the $\mathrm{x}$-ray wavelength of $\mathrm{Cu} K \alpha$ radiation $(1.54 \AA), \theta$ is the Bragg diffraction angle, and $\beta$ is the full width at half maximum of the respective diffraction peak. The larger the half-peak width, the smaller the grain size, i.e. more small grains are produced, indicating that a certain amount of Fe can inhibit the grain growth of zinc oxide.

EDS spectrum of as prepared Fe-doped $\mathrm{ZnO}$ thin films is shown in Figure 2, which reveals the atomic ratio of $\mathrm{Zn}$ and $\mathrm{O}$ in the products that is less than the stoichiometric ratio (1.2: 1), leading to the formation of oxygen vacancies. A relatively weak carbon and oxygen peaks in the spectrum probably originates from unavoidable surface contaminative carbon and surface-adsorption of oxygen on the surface of sample from exposure to air during sample preparing. After doping Fe atoms in $\mathrm{ZnO}$, the atomic ratio of $\mathrm{Zn}$ to $\mathrm{O}$ is decreased to 1.18:1,
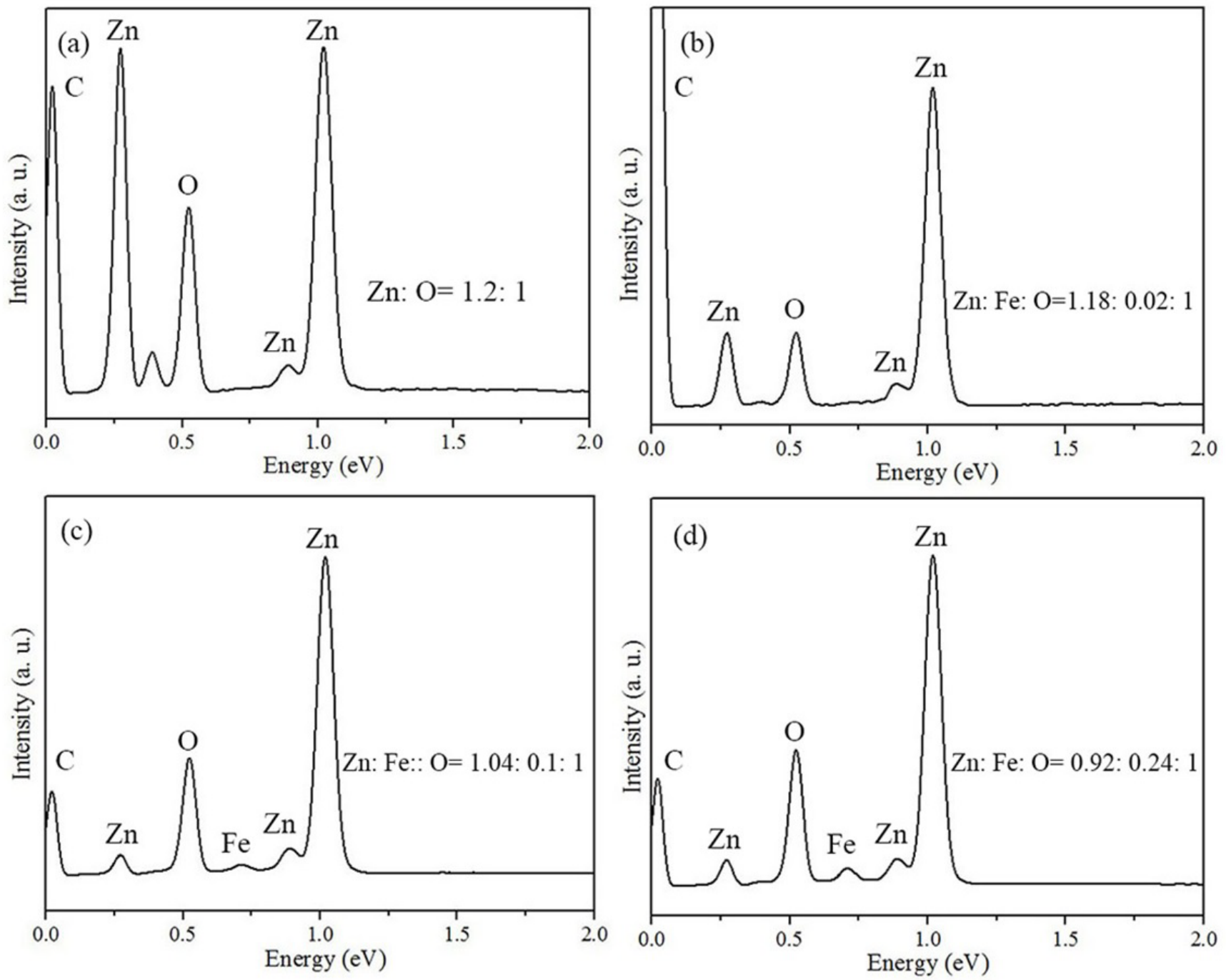

Figure 2: EDS of Fe-doped ZnO with different Fe contents: a) 0; b) 1\%; c) 5\%; d) $10 \%$. 

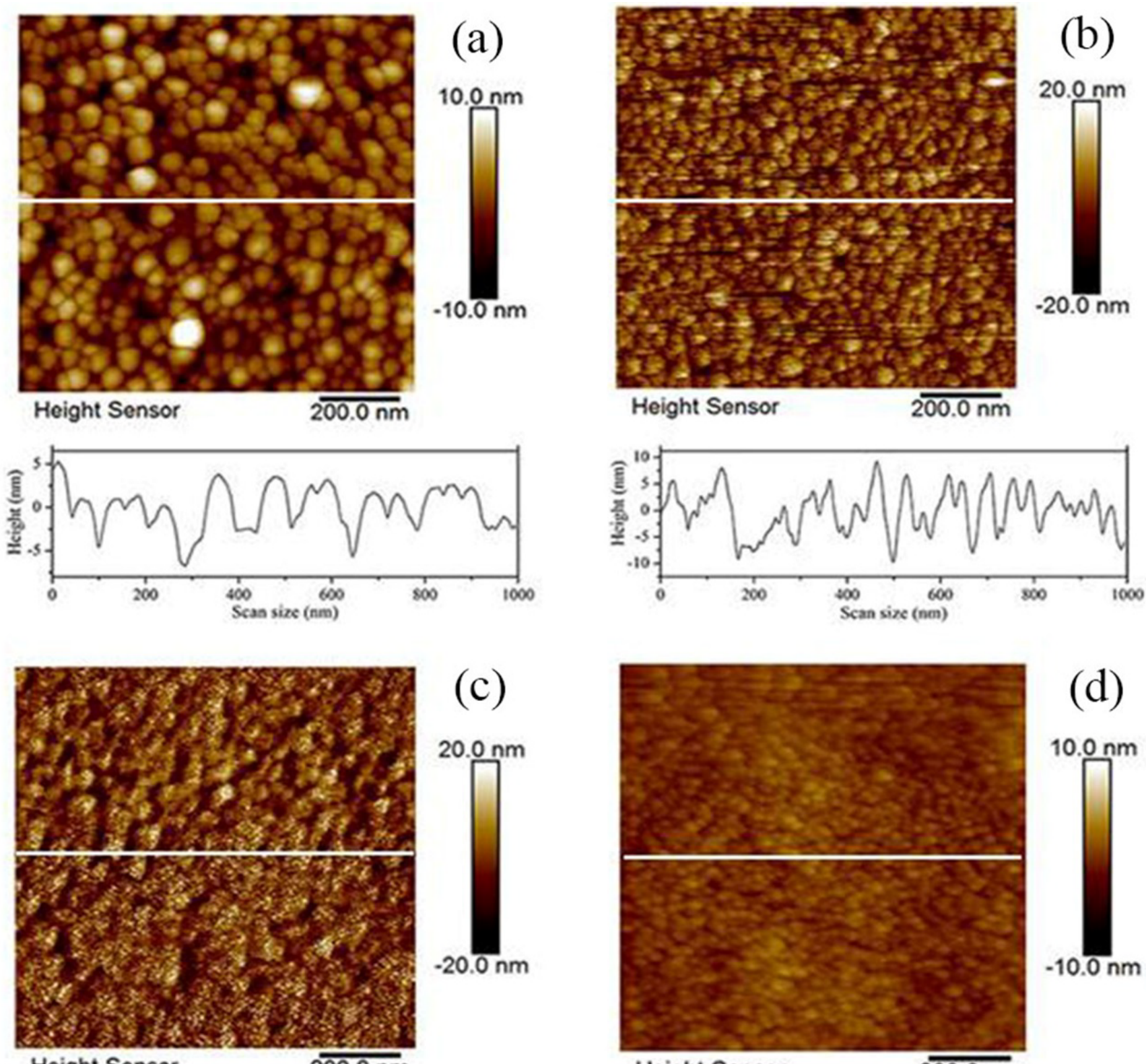

Height Sensor

$\overline{200.0 \mathrm{~nm}}$
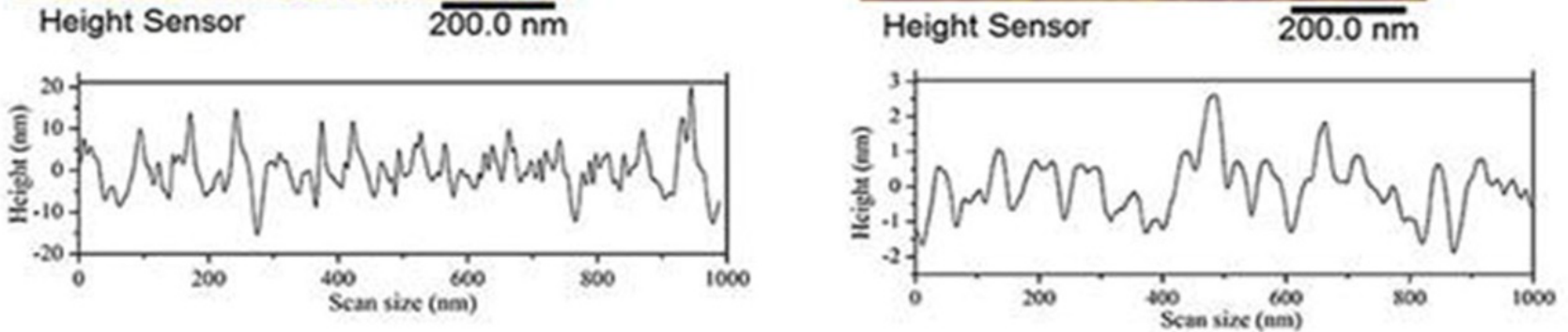

Figure 3: Two-dimensional (2D) AFM height images of Fe-doped ZnO thin films with different Fe contents: a) 0; b) $1 \%$; c) $5 \%$; d) $10 \%$.

1.04:1 and $0.92: 1$, indicating that some $\mathrm{Zn}$ atomic lattice is replaced by Fe atom to form solid solution, which is consistent to the XRD analysis.

Figure 3a, Figure 3b, Figure $3 c$ and Figure 3d show two-dimensional (2D) AFM surface height morphologies of $\mathrm{xFe}-\mathrm{ZnO}$ thin films $(\mathrm{x}=0,1,5$ and
$10 \mathrm{~mol} \%)$. The scanning area is $1 \mu \mathrm{m}^{2}$. Each film was composed of many fine $\mathrm{ZnO}$ particles. Obviously, with the increasing Fe dopant content from 0, 1, 5 to $10 \mathrm{~mol} \%$, the average grain size of $\mathrm{ZnO}$ was decreased from 40,30, $25 \mathrm{~nm}$ to $20 \mathrm{~nm}$, which is conformed to the XRD results. Under each AFM image, the section of the white line in the image was 
shown, indicating that the surface roughness was decreased after iron doping. However, in Figure 3d, excess Fe doping will increase the surface roughness due to the phase separation.

MFM is a kind of AFM, which scans magnetic samples with magnetic probes and detects the interaction between the probes and the samples to reconstruct the magnetic structure of the sample surface [14]. The samples were scanned by MFM with a scanning range of $1 \mu \mathrm{m}^{2}$. Antimonydoped silicon magnetic probe coated with cobaltchromium alloy was used. The resonance frequency and the elastic modulus of the probe was $75 \mathrm{kHz}$ and $2.8 \mathrm{~N} \mathrm{~m}^{-1}$, respectively. When the magnetic disturbance between the tip and the sample is not considered, the formula for calculating the magnetic force of the sample can be obtained [15], $F=-\nabla E=\mu_{0} \int \nabla\left(M_{\text {tip }} \cdot H_{\text {sample }}\right) d V_{\text {tip }}=\mu_{0} \int \nabla\left(M_{\text {sample }} \cdot H_{\text {tip }}\right) d V_{\text {sample }}$

That is, the convolution of the tip magnetic
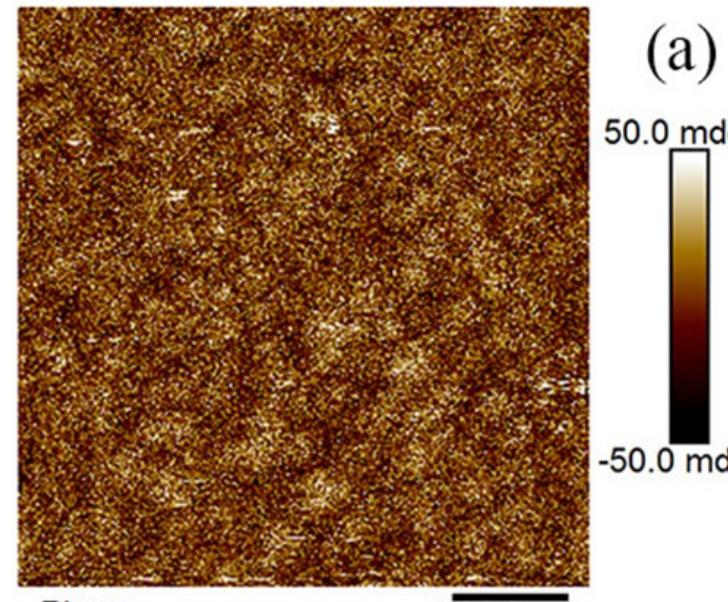

$\overline{200.0 \mathrm{~nm}}$
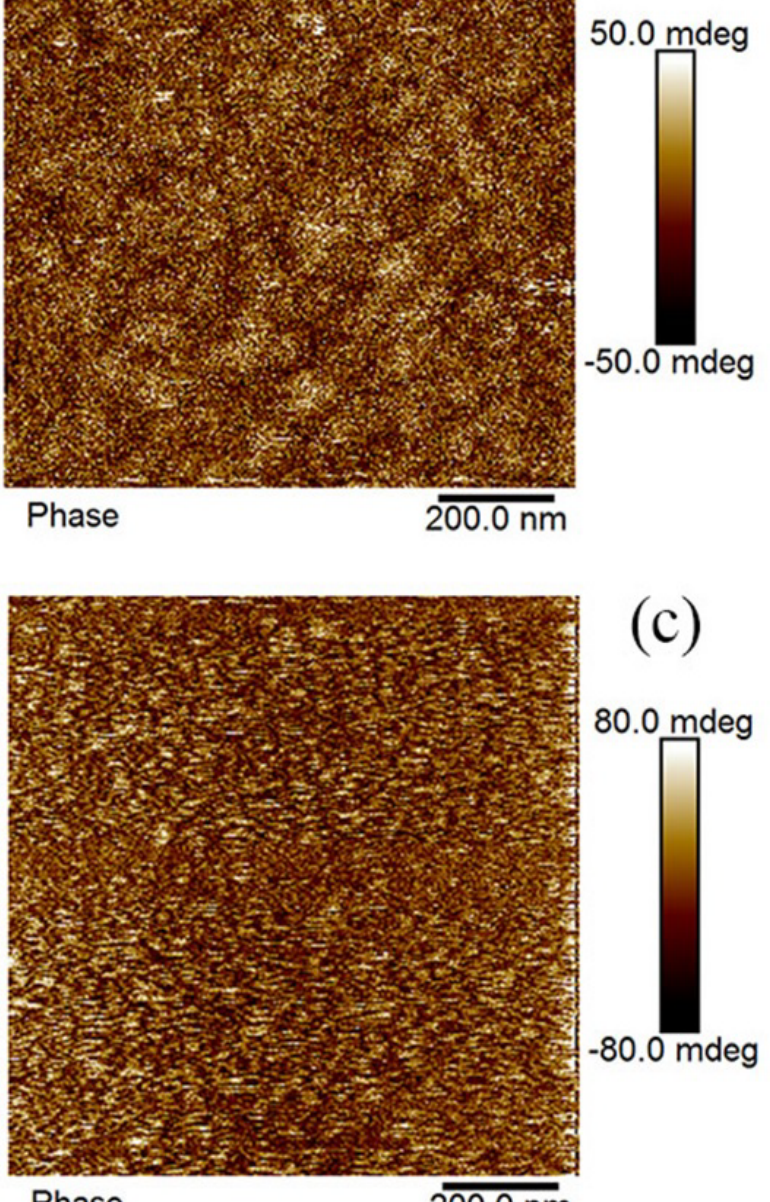

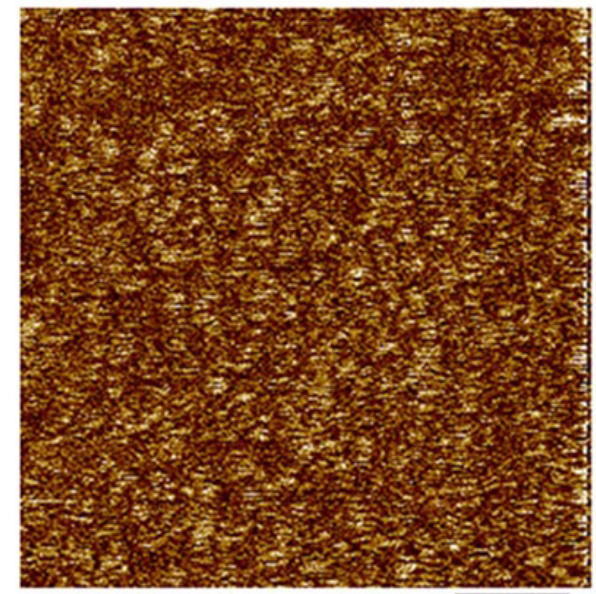

(b)

80.0 mdeg

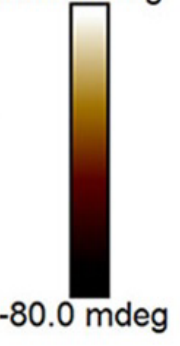

Phase

$\overline{200.0 \mathrm{~nm}}$

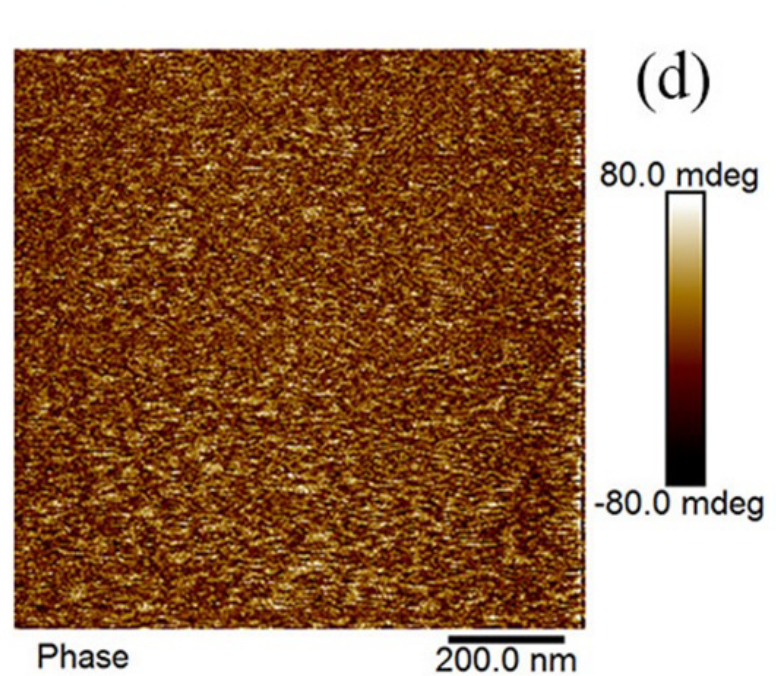

(d)

the vibration
cient, $k_{0}$ is the
frequency of
frequency of
is the driving
e MFM probe $\mathrm{H}_{\text {sample }}$ or the convolution of the sample moment $\mathrm{M}_{\text {sample }}$ with the tip stray magnetic field $\mathrm{H}_{\text {tip }}$. The corresponding MFM detection signal is proportional to the vertical component of the magnetic gradient, $\frac{\partial F}{\partial z}$. The vibration equation of the MFM probe is [16],

$m \frac{d^{2} z(t)}{d t^{2}}+m y \frac{d z(t)}{d t}+\left(k_{0}+\Delta k \cos \left(\omega_{m} t\right)\right) z(t)=F_{0} \cos \left(\omega_{c} t\right)$

Where $z$ is the coordinate of the vibration direction of the probe, $m$ is the effective mass of the probe, $\gamma$ is the damping coefficient, $k_{0}$ is the spring constant, $\Delta \mathrm{k}$ is the variation of effective spring coefficient, $\omega_{c}$ is the driving frequency of piezoelectric elements, $\omega_{m}$ is the frequency of high frequency magnetic field, $F_{0}$ is the driving can be considered as point magnetic charge $q_{t i p}$

Figure 4: MFM height images of Fe-doped ZnO thin films with different Fe contents: a) 0; b) 1\%; c) $5 \%$; d) $10 \%$. 
consequently,

$$
\Delta k=\frac{\partial F_{m}}{\partial z}=\frac{\partial}{\partial z} q_{t i p} H_{z} \cos \left(\omega_{m} t\right)
$$

Where $F_{m}$ is the alternating magnetic force between the probe and the sample, $\mathrm{H}_{2}$ is the amplitude of high frequency magnetic field. By adjusting the driving frequency of piezoelectric element $\omega_{c}$ to satisfy,

$$
\omega_{c}-\omega_{m}=\omega_{0}
$$

Where $\omega_{0}$ is the resonance frequency of the probe, as a result, the vibration signal of the probe is [17],

$$
z(t)=\frac{F_{0}}{m \gamma \omega_{0}} \sin \left(\omega_{c} t\right)+\frac{F_{0} \Delta k}{2\left(m \gamma \omega_{0}\right)^{2}} \cos \left[\left(\omega_{c}-\omega_{m}\right) t\right]
$$

Figure 4 is the MFM height images of $\mathrm{ZnO}$ thin films with different Fe content. Obviously, the intrinsic zinc oxide has no domain and magnetic moment distribution. After a certain amount of iron doping, the distribution of domain and magnetic moment is obtained. With the increase of iron doping, its magnetism tends to increase, the bright and dark fringes of domain are more clearly visible, indicating that a certain amount of iron doping can make the non-magnetic zinc oxide semiconductor become dilute magnetic semiconductor. However, when the iron doping content reaches $10 \%$, its magnetic properties decrease, which indicates that iron doping has a most suitable proportion and is less than $10 \%$.

\section{Conclusions}

In summary, a certain amount of iron doping can inhibit the growth of zinc oxide particles, and then grow more small crystal particles. With the increase of iron doping content, its magnetism tends to increase. A certain amount of iron doping can make non-magnetic zinc oxide semiconductor become dilute magnetic semiconductor, but the magnetism enhanced by iron doping is limited, and the upper limit is less than $10 \%$ of iron doping content. The surface roughness of the films increases with the increase of iron doping, because the stable solid solution cannot be formed due to the separation of two phases.

\section{Acknowledgements}

This work was financially supported by the Scientific and technological project of Henan province (182102210504), the national natural science foun- dation of China (51772256), the program for innovative research team (in science \& technology) in university of Henan province (19IRTSTHN026).

\section{References}

1. RA Torquato, SE Shirsath, RHGA Kiminami, ACFM Costa (2018) Synthesis and structural, magnetic characterization of nanocrystalline $\mathrm{Zn}_{1-x} \mathrm{Co}_{x} \mathrm{O}$ diluted magnetic semiconductors (DMS) synthesized by combustion reaction. Ceram Int 44: 4126-4131.

2. YD Zhang, LW Wang, LW Mi, FL Yang, Z Zheng (2011) Silica-controlled structure and optical properties of zinc oxide sol-gel thin films. J Mater Res 26: 882-888.

3. ZA Yunusov, SU Yuldashev, YH Kwon, DY Kim, SJ Lee, et al. (2018) Band gap engineering of $Z n M n O$ diluted magnetic semiconductor by alloying with ZnS. J Mag Mag Mater 446: 206-209.

4. KY Salkar, RB Tangsali, RS Gad, U Subramanian (2019) Preparation characterization and magnetic properties of $\mathrm{Zn}_{(1-x)} \mathrm{Co}_{x} \mathrm{O}$ nanoparticle Dilute magnetic semiconductors. Superlat Microstruct 126: 158-173.

5. C Belkhaoui, N Mzabi, H Smaoui (2019) Investigations on structural, optical and dielectric properties of $\mathrm{Mn}$ doped $\mathrm{ZnO}$ nanoparticles synthesized by coprecipitation method. Mater Res Bull 111: 70-79.

6. P Obreja, D Cristea, A Dinescu, C Romanitan (2019) Influence of surface substrates on the properties of ZnO nanowires synthesized by hydrothermal method. Appl Surf Sci 463: 1117-1123.

7. SBose, D Sanyal (2018) Synthesis and characterization of $\mathrm{ZnO}$ microfiber by electrospinning technique. Mater Today: proceedings 5: 9860-9865.

8. R Nasser, H Elhouihet (2018) Production of acceptor complexes in sol-gel $\mathrm{ZnO}$ thin films by Sb doping. J Lumines 196: 11-19.

9. CBS Valentin, RLS Silva, P Banerjee, A Franco (2019) Investigation of Fe-doped room temperature dilute ma gnetic $\mathrm{ZnO}$ semiconductors. Mater Sci Semiconduct Proces 96: 122-126.

10.XF Jia, QY Hou, ZC Xu, LF Qu (2018) Effect of Ce doping on the magnetic and optical properties of $\mathrm{ZnO}$ by the first principle. J Mag \& Mag Mater 465: 128-135.

11.KP Shinde, RC Pawar, BB Sinha, HS Kim, SS Oh, et al. (2014) Optical and magnetic properties of Ni doped ZnO planetary ball milled nanopowder synthesized by co-precipitation. Ceram Int 40: 16799-16804.

12.C Valentin, R Silva, P Banerjee, A Franco (2019) Investigation of Fe-doped room temperature dilute magnetic ZnO semiconductors. Mater Sci Semiconduct Processing 96: 122-126. 
13.YD Zhang, HM Jia, PJ Li, FL Yang, Z Zheng (2011) Influence of glucose on the structural and optical properties of $\mathrm{ZnO}$ thin films prepared by sol-gel method. Opt Commun 284: 236-239.

14.MV Makarova, Y Akaishi, T Ikarshi, KS Rao, S Yoshimura, et al. (2019) Alternating Magnetic Force Microscopy: Effect of $\mathrm{Si}$ doping on the temporal performance degradation of amorphous $\mathrm{FeCoB}$ magnetic tips. J Mag \& Mag Mater 471: 209-214.

15.I Utke, P Hoffmann, R Berger (2002) High-resolution magnetic Co supertips grown by a focused electron beam. Appl Phys Lett 80: 4792.

16.SL Tomlison, AN Farley (1997) The magnetic force microscopy and its capability for nanomagnetic studies. J Appl Phys 81: 5029.

17.SJL Vellekoop, L Abelmann, S Porthun, JC Lodder, JJ Miles (1999) Calculation of playback signals from MFM images using transfer functions. J Mag \& Mag Mater 193: 474-478. 